\title{
Design and Implementation of Android IT Job Alliance System
}

\author{
Hai Wang ${ }^{1, a}$, Lan Huang ${ }^{2, b}$, Wuchao $\mathrm{Li}^{3, \mathrm{c}}$, Wei Luo ${ }^{4, \mathrm{~d}}$ \\ 1,2,3,4 Software College of Jilin University, Changchun 130012, China \\ ${ }^{2}$ Symbol Computation and Knowledge Engineering of Ministry of Education, Jilin University, Changchun \\ 130012, China \\ a13134484667@163.com \\ bhuanglan@jlu.edu.cn \\ c643101093@qq.com \\ d171339995@qq.com
}

Keywords: Android, IT, Job Guidance, C/S Architecture, HTTP, JSON

\begin{abstract}
In recent years, as the intelligent terminals developed rapidly, the combination of intelligent terminals and Internet has been an evident trend of information development and utility software. Based on the kernel of Linux V2.6, Android is the representative of mobile terminal, which is an open operating system, released by Google at November 2007, possessing broad application and development prospect. The "IT Job Alliance" system is based on Android operating system, aiming at relieving the situation that the undergraduates study aimlessly and poorly reach the corporation's standards after graduation. There are four parts in this system, including "Recruit Information”, “Job Market Guidance”, “Interaction” and "University and Company”. Through the "thin" client C/S (Client/Server) architecture, the system gains considerable expansibility and maintainability.
\end{abstract}

\section{Introduction}

As the industry developed at top speed, Internet is talent-intensive and information changes quickly. The undergraduates study with a bit aimless as they are far away from companies and job market. As a result, there are large disparities between graduates and the employee's standard company expected. And the situation of Internet job market doesn't have a great improvement till now. With the continuous development of micro processor's performance and the further improvement of embedded operating system, the intelligent terminals have covered everywhere of our life with an unstoppable momentum. Smart phone has been the indispensable part of our daily life, study, work and communication.

At present, a series of similar products based on smart phone only provide the recruitment platform at home and abroad. But doesn't contact companies and universities to give guidance to undergraduates or form a virtuous circle. So the appearance of an application based on Android operating system and aimed at improving the environment of Internet job market and the poor situation of undergraduates' studying is inevitable. It not only provides a platform for recruitment but contacts the corporations, schools and schoolmates to give a comprehensive guidance for undergraduates.

\section{Software Platform--Android Operating System}

Android, an open source operating system based on Linux V2.6 kernel, was released by Google in November, 2007. Its openness, hardware richness and development convenience make it occupied $81.5 \%$ of the market share of smart phone operating system in the first quarter of 2015. In addition, it's also one of most popular development platforms for developers.

Android operating system structure: 


\begin{tabular}{|c|c|}
\hline \multicolumn{2}{|c|}{ Applications } \\
\hline \multicolumn{2}{|c|}{ Application Framework } \\
\hline Libraries & Android Runtime \\
\hline \multicolumn{2}{|c|}{ Linux Kernel } \\
\hline
\end{tabular}

Fig. 1Android operating system structure

Applications: Java programs run on virtual machine.

Application Framework: Some API frameworks used by the core applications released by Google. The developers could use these API to develop their applications.

Libraries and Android Runtime: It consists several C/C++ libraries which can be used by vary components of Android. And provides severs for developers through Application Framework.

Dalvik virtual machine works on Android operating system and every java program should run on it. It is suitable for mobile terminal because it doesn’t require fast processing speed or lots of memory.

\section{Designs and Implementation of the System}

Designs of the System Architecture. "IT Job Alliance” system uses C/S (Client/Server) architecture. With the restriction of storage space and processing capacity, the "thin" client C/S architecture meets the developing requirements of mobile phone.

As the Client, the mobile phone realizes the interaction with users including the entry, display and simple process of data.

The Server contains Business Logic Layer and Data layer. The Business Logic Layer is realized with Servlets and some auxiliary classes. As the bridge between View Layer and Data layer, the Business Logic Layer mainly completes data analysis and processing. The Data layer is composed of several DAO classes which mainly completes data access.

The Necessity of this System Used the C/S Architecture. The development mode of the combination of the mobile terminals and Internet has been matured. The complicated data processing tasks are distributed to Server node. The portable Client only needs to receive and display the information. As well, it could do some simple data processing sometimes. This can greatly improved Client's response speed and data handing capacity.

The hierarchical structure separates the data display, processing and storage in a proper way which is advantageous to the maintenance, upgrades and safety of the system.

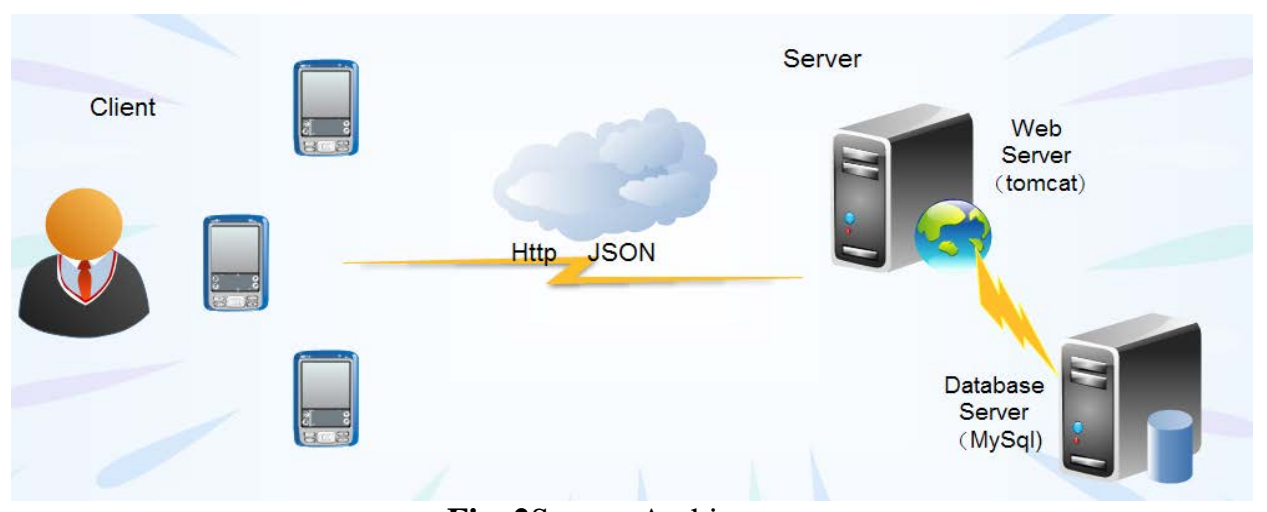

Fig. 2System Architecture

The Implementation of the Client. The system based on Android operating system which provides the rich and powerful components with that the viewer has a good user experience. The Client sends the data from users to Server to process and then the result returned to Client to show. We use the JSON (JavaScript Object Notation) as the data format to exchange information between Client and Server. As a lightweight data-interchange format, JSON uses the text format which is completely independent from different programming languages. The data format of JSON is simple and 
readable. In addition, it not only supports several programming languages but has a satisfactory transmission and parsing speed $[3,4,6]$.

The Implementation of the Server-side. The system's Server is Tomcat 8.0.24 of Apache. The Server-side is divided into the Business Logic Layer and Data Layer.

The Business Logic Layer: The system uses HTTP as the communication mode between Clients and Server. The data is encapsulated into the JSON data format in the Client and transmitted to the Servlet of the Business Logic Layer [2, 7]. Then the Servlet and some auxiliary classes parse and process the data and call the corresponding interfaces of the DAO classes. The Data Layer gets the data and returns it to the Business Logic Layer which makes the further data process and encapsulation. Finally, the information is returned to Client to show.

The Data Layer: The data layer accesses data from database according to the requirement of Business Logic Layer. The database the system used is Oracle's opening relational database--MySql which is small in scale, fast in speed and low in cost. It can either as a separate application applied in the C/S network environment or embedded into other software as a library. And it is flexible for the multilingual support.

\section{Design and Implementation of the System's Function}

\subsection{System Function Structure Diagram}

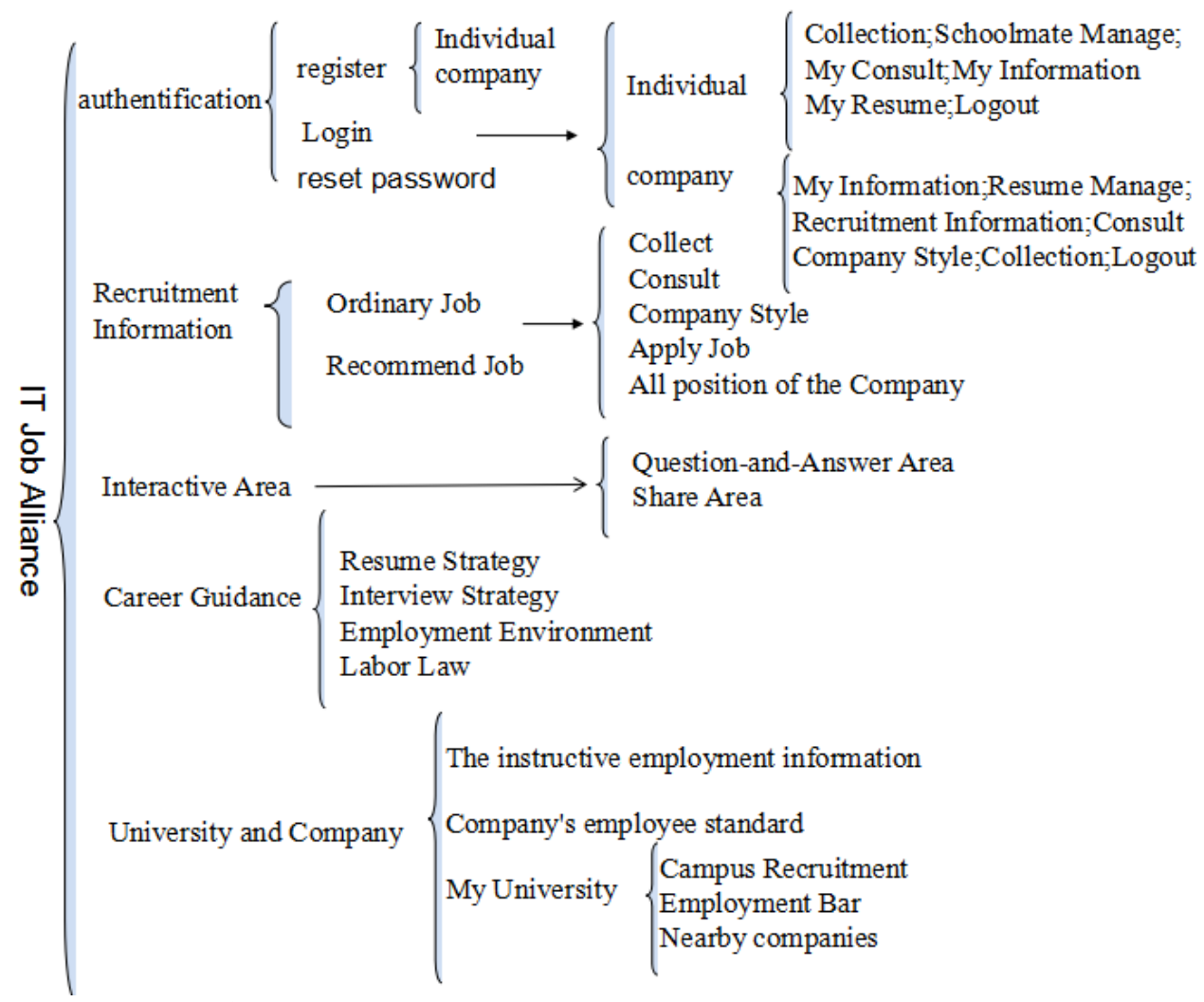

Fig. 3System Function Structure Diagram

\subsection{The Implementation of Each System Module}

Register and Login Module. Users are divided into individual and company when they register and login. The registration information will be checked strictly such as the format and availability of the phone number. Password stored after encrypted by MD5 algorithm. In order to avoid the boring process of login, the system uses the SharedPreferences provided by Android to realize the "Remember Password" function [1]. The system supports a variety of ways to login such as by username, binding phone number and email address. Through SMS provided by the third part, the system realizes the "Reset Password" function. 
Recruitment Information Module. The companies publish the recruitment information at "Company Center". The job hunters could screen the information from several aspects, for instance, working place, salary, the enterprise property and so on. For each of the recruitment information, the user can consult, collect and apply for. Furthermore, the company style and all the positions published by the company could be access from here. The system gets the users' location after permission through the Location SDK provided by Baidu lnc [8]. Accordingly, the local positions will be recommended at first. After the match between the "My Ideal Position" at "Individual Center" and the positions published, the top $\mathrm{N}$ positions will be recommended to users.

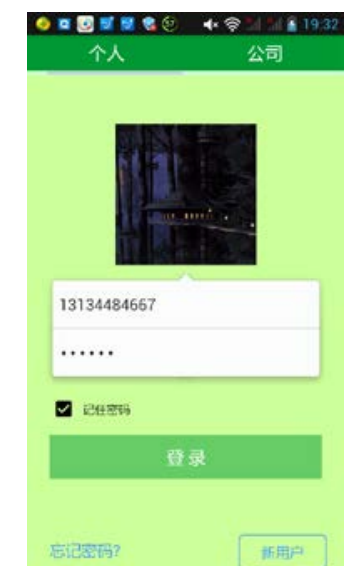

Fig. 4Individual Login Page

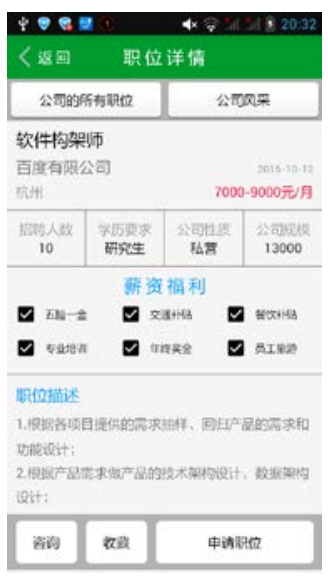

Fig. 5The recruitment Information Page

Interaction Module. The Interaction Module is divided into Question-and-Answer area and Share area.

Question-and-Answer area: All the users can publish their own questions or answer questions raised by others. Moreover, for each question, the user can praise and collect. The display of information is sorted according to the date of descending. Share area: Users can publish their own experience, professional knowledge and so on.

In the Interaction Module, you could concern the publisher. After concerned successfully, you can leave a message to him and query his interaction before.

Career Guidance Module. “Career Guidance” provides a platform for the job seekers and incumbents to have an in-depth know of the job market. All the information of this module is acquired from the Internet through the simple web crawler technology. Firstly, the system provides several source URL (some large recruitment websites' Career Guidance's URL) from which getting the useful information and available URL after parsing the source code. While the process would be stopped when the total number of the information reaches the upper limit. The HTML parsing tool the system used is HtmlParser library which is written in pure Java programming language and independent to other Java library. The capacity of accessing and parsing HTML with a high speed make itself a good tool. Certainly, the users can also collect the articles which he interested in [5].

University and Company Module. The "University and Company" mainly contacts the universities, companies and undergraduates to have more interactive and cooperation.

There are two sources of the instructive employment information. One is getting from the Internet, the other is the statistic result of the employment completed in this system.

The registered companies could publish employees' standards such as the professional skills and all kinds of certificates of different positions which is benefit to the targeted study of undergraduates.

My University: This is a platform to active with their own schoolmates. Actually, the companies could publish the information of campus recruitment at "Campus Recruitment” module. It is useful that the frequent communication among companies, universities and students about employment for students to know more about employment At Employment Bar. In addition, searching the IT companies near the users through the Location SDK provided by Baidu lnc provides the potential 
opportunities of internship [8].

\section{Conclusions}

The paper describes the design and implementation of "IT Job Alliance" system, a platform based on Android operating system for companies, universities and undergraduates to improve the condition of students' aimless study and the unbalance job market. According to the features of smart phone and Internet, the system adopts the "thin" Client C/S architecture. The combination of the portability of smart phone and extensive of Internet makes the great use of the both advantages. In addition, the three layer structure of the system is benefit to develop each part individually and the maintenance, upgrade, and transplantation of the system. After the rapid development of Internet industry, it is becoming more and more apparent that the rapid iteration of the information and the heavy demand for the talents. While the contradiction between it and the present situation of job market will have become increasingly obvious. The "IT Job Alliance" contacts the companies, universities and students to give the comprehensive guidance to undergraduates starting from recruitment and university's teaching. As a result, the measure fundamentally eases the current situation and forms a virtuous circle.

\section{References}

1. G. Li. Crazy Android Notes (the second edition, 2013.03), [M]. Beijing: Electronic Industry Press.

2. W. Chen, Y. Z. Guo. Deep Understanding of the Android Network Programming (the first edition, 2014.09), [M]. Beijing: China Machine Press.

3. R. Meier. Professional Android 4 Application Development (2013.04), [M]. J. W. Yu, K. Zhao. Beijing: Tsinghua University Press.

4. E. R. Harold. Java Network Programming (the third edition, 2005.11), [M]. J. T. Zhu, J. Lin, Beijing: China Electric Power Press.

5. M. L. Zhao. Design and Research of Network Spider; [D]. Sichuan: University of Electronic Science of Technology of China (2013).

6. Z. M. Quan, X. Y. Peng. An Efficient and Safe Way of Accessing Remote Database by JSON in Android System; [J]. Journal of Shaoguan University.Nature Science, 32, 16-20 (2011).

7. Y. S. Wang. The development pattern of HTTP communication of Android APP; [J]. Computer Programming Skills \& Maintenance, 19, 41-47 (2014).

8. B. Zhang, S. M. Zhao. Development and Research of Baidu Map Based on Android System; [J]. Software Guide, 14, 96-98(2015). 